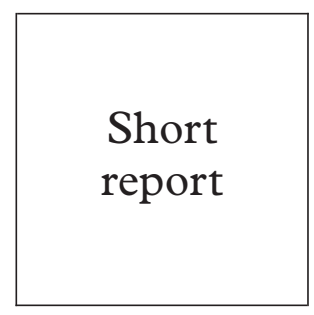

\title{
Predictors of time spent on partner notification in four US sites
}

\author{
Beth A Macke, Michael H Hennessy, Mary McFarlane
}

Objective: To identify determinants of time spent on partner notification clients in four STD programmes in the United States.

Methods: 11 disease intervention specialists (DIS) in each of three urban sites $(n=33)$ and seven DIS in one rural site recorded their activities and clients for 14 working days. The total amount of time for partner notification activities was computed for each client. Data were analysed using random effects regression.

Results: Across sites, 429 of 2506 (37.4\%) recorded hours were spent on partner notification (PN) activities with 1148 clients. Client type, STD diagnosis, outcome, demographic characteristics, mileage, and study site explained $33.7 \%$ of the variance in the total time spent on partner notification clients. Clients who took significantly more time than the reference case included those who were both contacts and original patients, HIV/AIDS clients, non-primary and secondary (P\&S) syphilis clients, STD clients who were infected and treated, and clients for whom travel was necessary. Demographic characteristics of both client and worker were not associated with the time spent on partner notification.

Conclusions: These data document the labour intensive nature of partner notification, especially for HIV and non-P\&S syphilis clients. STD programmes that have a higher number of these clients are probably dedicating more resources to partner notification. More research is needed on additional predictors so that programmes can better understand and allocate staff and financial resources to partner notification activities.

(Sex Transm Inf 2000;76:371-374)

Keywords: partner notification; STD; HIV

\section{Introduction}

Provider assisted partner notification (PN), the process by which a partner of a person known to have a sexually transmitted disease (STD) is informed by a health official that he or she has been exposed to an STD and encouraged to seek medical evaluation, has been a public health strategy for the control of syphilis transmission since the $1930 \mathrm{~s},{ }^{1}$ and has been used more recently as a control strategy for $\mathrm{HIV}^{23}$ gonorrhoea, ${ }^{4}$ and chlamydia. ${ }^{45}$ In a previous analysis, the types of activities associated with $\mathrm{PN}$ and the time spent on those activities were described. ${ }^{6}$ The current analysis examines predictors of the amount of time spent on a PN client, including client type, disease, demographic characteristics, or outcome of the client. With a better understanding of current resource allocation, programmes will be able to better anticipate and meet future needs.

\section{Methods}

The site selection, data collection methods, and the descriptive results of this exploratory study are available elsewhere. ${ }^{6}$ In summary, four sites were selected based on their representation of different geographic regions, a variety of practice settings, and different levels of disease morbidity. Eleven disease intervention specialists (DIS) in each of the three urban sites $(n=33)$ and seven DIS in the rural site documented their PN activities using methods that minimised work interruption, response errors, and reporting biases throughout the working day for 14 consecutive working days. This study was exempted from human subjects review.

VARIABLES

Dependent variable

The dependent variable is the average total number of minutes spent on each client summed across all PN activities. The PN activities included interviewing, record searches, phone calls, field visits, treatment verification, provider visits, clinic services, and the documentation of these activities; these activities have been described in detail elsewhere. $^{6}$

\section{Predictors}

Clients were classified into four types: (1) an original (index) patient (that is, an infected person from whom contacts are elicited); (2) a contact of the original patient (that is, a sex or needle sharing partner who is thought to have been exposed); (3) a client who was both an original patient and a contact of another patient; and (4) a client not classified in any of the previous three categories because of insufficient data. With respect to disease, clients were classified into five disease types, according to the disease with which they were associated: (1) chlamydia, (2) gonorrhoea, (3) HIV infection, (4) primary and secondary (P\&S) syphilis, and (5) other (non-primary and secondary) syphilis. While clients may have been diagnosed with other STDs, data were not collected on these STDs since they do not rou- 
Table 1 Description of variables and predictors of total time (minutes) spent on PN clients using generalised least squares regression $\left(R^{2}=33.7 ; n=1131\right)$

\begin{tabular}{|c|c|c|c|c|c|}
\hline Variable & Percentage* & Coefficient & Standard error & $t$ Ratiof & Probability \\
\hline Intercept & & 64.77 & 15.28 & 4.23 & 0.00 \\
\hline \multicolumn{6}{|l|}{ Client type } \\
\hline Contact & 34.6 & -10.88 & 2.76 & -3.99 & 0.00 \\
\hline Both contact and original patient & 3.6 & 74.29 & 6.40 & 11.62 & 0.00 \\
\hline Unclassified & 5.8 & -40.31 & 5.42 & -7.44 & 0.00 \\
\hline (Reference $=$ original patient $)$ & 56.0 & & & & \\
\hline \multicolumn{6}{|l|}{ STD } \\
\hline Gonorrhoa & 25.5 & -6.58 & 4.17 & -1.57 & 0.11 \\
\hline HIV & 10.1 & 16.95 & 6.33 & 2.67 & 0.00 \\
\hline P\&S syphilis & 6.5 & 5.17 & 5.79 & 0.89 & 0.37 \\
\hline Other syphilis & 39.9 & 14.01 & 3.91 & 3.58 & 0.00 \\
\hline (Reference $=$ chlamydia $)$ & 18.0 & & & & \\
\hline \multicolumn{6}{|l|}{ Outcome } \\
\hline Prophylactic treatment & 7.6 & 8.64 & 7.99 & 1.08 & 0.28 \\
\hline Infected and treated & 31.2 & 15.69 & 7.06 & 2.23 & 0.03 \\
\hline Previously treated & 17.1 & 13.37 & 7.27 & 1.84 & 0.07 \\
\hline Refused treatment & 3.4 & 15.67 & 8.84 & 1.77 & 0.08 \\
\hline Unable to locate & 9.5 & 8.48 & 7.59 & 1.11 & 0.26 \\
\hline New positive (HIV) & 1.1 & 59.08 & 13.17 & 4.49 & 0.00 \\
\hline Previously known positive (HIV) & 2.2 & 18.83 & 10.91 & 1.72 & 0.08 \\
\hline Negative/not infected & 6.5 & 5.67 & 7.97 & 0.71 & 0.47 \\
\hline Other & 1.5 & 8.29 & 11.28 & 0.74 & 0.46 \\
\hline Missing & 18.3 & 11.70 & 7.34 & 1.59 & 0.11 \\
\hline (Reference $=$ administrative closure $)$ & 3.1 & & & & \\
\hline \multicolumn{6}{|l|}{ Client characteristics } \\
\hline Age (years) & & -0.15 & 0.13 & -1.14 & 0.26 \\
\hline \multicolumn{6}{|l|}{ Race/ethnicity } \\
\hline White & 6.4 & -0.92 & 6.03 & -0.15 & 0.88 \\
\hline Hispanic & 9.7 & 0.95 & 5.75 & 0.17 & 0.87 \\
\hline Other & 12.8 & -2.47 & 5.55 & -0.45 & 0.65 \\
\hline$($ Reference $=$ black $)$ & 71.1 & & & & \\
\hline Male (Reference $=$ female $)$ & 45.7 & 0.35 & 3.23 & 0.11 & 0.91 \\
\hline \multicolumn{6}{|l|}{ DIS characteristics } \\
\hline \multicolumn{6}{|l|}{ Race/ethnicity } \\
\hline White & 18.8 & 12.63 & 8.84 & 1.43 & 0.15 \\
\hline Asian & 3.8 & -8.75 & 14.27 & -0.61 & 0.54 \\
\hline Hispanic & 2.5 & 22.41 & 19.42 & 1.16 & 0.25 \\
\hline Other & 2.5 & 5.47 & 19.63 & 0.27 & 0.78 \\
\hline$($ Reference $=$ black $)$ & 72.4 & & & & \\
\hline Male $($ Reference $=$ female $)$ & 47.8 & 7.77 & 6.74 & 1.52 & 0.25 \\
\hline Years of experience & & -1.08 & 0.82 & -1.31 & 0.19 \\
\hline \multicolumn{6}{|l|}{ DIS/client match } \\
\hline Sex match (male) & 19.3 & -2.10 & 4.59 & -0.46 & 0.64 \\
\hline Ethnicity match (black) & 58.4 & -4.58 & 5.41 & -0.85 & 0.40 \\
\hline Miles driven per client & & 0.51 & 0.13 & 3.83 & 0.00 \\
\hline Percentage of clients with mileage $>0$ & 26.2 & & & & \\
\hline \multicolumn{6}{|l|}{ Study site } \\
\hline Northeast & 33.3 & -42.02 & 10.77 & -3.93 & 0.00 \\
\hline West & 22.0 & -22.21 & 12.85 & -1.73 & 0.08 \\
\hline Midwest & 31.1 & -18.10 & 11.62 & -1.56 & 0.12 \\
\hline (Reference $=$ south $)$ & 13.5 & & & & \\
\hline
\end{tabular}

${ }^{\star}$ All percentages in column 1 use the total patient population as the denominator (which includes original or index patients as well as contacts to index patients). For example, $18.8 \%$ of the PN clients were managed by white DIS and $72.4 \%$ of the clients were managed by black DIS.

†Statistically significant $t$ ratios (>1.96) are in bold type.

Note: The intercept of 64.77 minutes represents the average total time spent on a black female original patient in the South site who has a diagnosis of chlamydia and an outcome of administrative closure; because the interaction term matches black clients with black workers, the actual number of minutes spent on the referent client is 60.19 (64.77 minus 4.58) minutes. The average total time spent for clients with other characteristics can be calculated by adding the coefficients (or subtracting if a negative coefficient) for the respective characteristic.

tinely involve PN. Outcomes for PN clients, listed in table 1, were assigned when either: (1) the client has been located, referred, medically evaluated (and interviewed, if infected) and the investigation is closed or (2) the client cannot be located, is out of jurisdiction, or refuses counseling and testing. Different outcomes may have important implications for the total time spent on a case. Administrative closure, the reference category, means that there was insufficient information to begin the investigation.

For the client, demographic characteristics included age, sex, and self identified ethnic status documented as black, Hispanic, other, or white. The age, sex, and self identified ethnic status reported here are of the 36 DIS but are described in relation to the clients. We also investigate the predictive power of the match between the DIS and the client on the basis of both sex and ethnicity. However, because of the low numbers of non-black DIS, we only examined the effect of ethnicity matching for black clients and black DIS.

$\mathrm{PN}$ activities often require travel during the case investigation process. For these cases, we include the total number of miles reported for the case investigation. Finally, we include dummy variables to indicate the study site in order to examine other general factors that may affect total time on a case but are not captured by the previously described variables.

\section{ANALYSIS}

All regression analyses were performed with the programmes The Econometrics Toolkit ${ }^{7}$ and LIMDEP $^{8}$ using random effects regression analysis. This generalised least squares method adjusts for the non-independence of the observations at the client level ${ }^{9}{ }^{10}$ that results when 
clients in the same site have the same DIS in charge of their case management. The coefficients presented in the results that follow represent the number of minutes (either more if the coefficient is positive, or less if negative) spent on clients with certain characteristics compared with the referent client. Associated with each coefficient is a $t$ ratio; any $t$ ratio greater than 1.96 in absolute value defines statistically significant association with the number of minutes spent on a client with that characteristic. Data collected from four DIS who were unable to complete the entire study and 17 patients with unknown age were excluded before analysis to ensure the same number of observations were used to estimate every variable.

\section{Results}

The percentage distributions for all of the variables used in the analysis are listed in the first column of table 1 . The average total number of minutes spent on each client summed across all PN activities was a mean of 46.09 (SD 47.98) minutes. The majority of clients were original patients $(56 \%)$ and nearly half were associated with some form of syphilis (46\%). The most common outcome was infected and treated $(31 \%)$. Over two thirds of clients $(76 \%)$ and DIS (72\%) identified themselves as black. The mean age of the DIS was 29.5 years (10.1 years). The mean years of experience was 5.3 (5.0) years. About $19 \%$ of all the patients were assigned to a DIS of the same sex and $58.4 \%$ of all patients were assigned a DIS who matched the patients' ethnicity. Note that, of only black patients $(n=815), 82 \%$ were matched with a black DIS. The mean mileage for cases which involved some travel was 11.87 (15.93) miles. Around one third of the clients came from both the northeast and midwest sites, followed by the west $(22 \%)$ and the south (14\%).

The results of the generalised least squares regression are presented in table 1 (columns $2-4)$. These variables explain $34 \%$ of the variance in time spent on a PN client. The intercept is 65 minutes and represents the PN activities for a black female original patient in the south site who has a diagnosis of chlamydia and an outcome of administrative closure; because the interaction term matches black clients with black workers, the true total for the referent client is 60 (65 minus 5) minutes.

Client type contributes the single largest increment to total time spent on PN clients. Compared with the time spent on original patients, significantly less time was spent on contacts (11 minutes less than original patients) and on unclassified clients (40 minutes less) whereas clients classified as both contacts and original patients required significantly more time (74 minutes). Clients diagnosed with other syphilis or with HIV required significantly more time, 14 minutes and 17 minutes more, respectively, than those diagnosed with chlamydia. Cases classified as "newly HIV positive" required nearly an hour (59 minutes) longer and cases that resulted in the location and treatment of people who were infected with an STD required significantly more DIS time (16 minutes) than cases with an outcome of administrative closure. None of the demographic characteristics was related to the amount of time spent by DIS on PN clients. Each mile contributed a small (30 seconds per mile) but significant amount of additional time spent on a case. Finally, the northeast site spent nearly 4 minutes less per client.

\section{Discussion}

The goal of this exploratory study was to document the process of PN in four public health settings and identify the predictors of the amount of time spent on that process. While these four sites vary with respect to demographics and disease morbidity, they do not represent all PN in the United States, nor do the 36 DIS represent the work done by all DIS. In addition, because this study was conducted for only a 3 week period, activities with some clients had already begun before the study, while activities on other clients had been started but not completed by the time the study ended, so it is possible to have missed some activities. The results show that PN client type, STD, outcome, and mileage were directly related to the total time spent on clients. The wide variances associated with both the dependent and the predictor variables suggest a cautious interpretation. Because these variables explained only one third of the variance in time spent on PN clients, research is needed to explore other influences on PN activity at the community, programme, worker, and client levels.

Clients who are both contacts and original patients take more than twice the time of either contacts or original patients separately. Newly diagnosed HIV clients require the most resources, followed by non-primary and secondary syphilis. PN programmes that serve a greater number of HIV and non-primary and secondary syphilis clients will probably require more resources per client.

Only the northeast site spent significantly less time (42 minutes) per client than the reference site (south). One explanation is that in contrast with the other three sites, the northeast site kept all records on a secure and efficient computer system which enabled more than one worker to access information about PN clients instantly. When a contact called the health department in response to a confidential referral letter, any worker who received the call could look up the case and give the contact information about the exposure and counsel the contact. For the other three sites, only the DIS actually assigned to the case could provide this information. If that DIS was busy or out of the office, the contact would have to wait and, in a number of cases, phone or field "tag" could go on for days, resulting in a delay in notification and, ultimately, a delay in evaluation and treatment of the partner.

These results suggest that technology may have a role in increasing the efficiency of PN. As these data can assist PN programmes in understanding and improving resource allocation, programmes should consider conducting similar studies in their own areas. 
The authors thank the disease intervention specialists (DIS) and their supervisors at the four sites for their cooperation and dedication to this project.

Contributors: BAM designed the study and collected the data; $\mathrm{MHH}$ and MM analysed the data and assisted with the writing of the manuscript.

1 Parran T. Shadow on the land: syphilis. New York: Reynal and Hitchcock, 1937.

2 Landis SE, Schoenbach VJ, Weber DJ. Results of a randomized trial of partner notification in cases of HIV infection in North Carolina. N Engl F Med 1992;326:101-6.

3 Wykoff RF, Jones JL, Longshore ST, et al. Notification of the sex and needle-sharing partners of individuals with human immunodeficiency virus in rural South Carolina: 30-month experience. Sex Transm Dis 1991;18:217-22.

4 Alary M, Joly J, Poulin C. Gonorrhea and chlamydial infection: comparison of contact tracing performed by phy- sicians or by a specialized service. Can $\mathcal{F}$ Public Health $1991 ; 82: 132-4$

5 Katz BP, Danos S, Quinn TS, et al. Efficiency and cost-effectiveness of field follow-up for patients with chlamydia trachomatis infection in a sexually transmitted diseases clinic. Sex Transm Dis 1988;15:11-16.

6 Macke BA, Hennessy MH, McFarlane M, et al. Partner notification in the real world: a four site time-motion study. Sex Transm Dis 1998;25:561-8.

7 Greene W. The Econometrics Toolkit: Version 3.0. New York: Econometric Software, 1992.

8 Greene W. LIMDEP Version 6.0 New York: Econometric Software, 1992

9 Donner A. A regression approach to the analysis of data arising from cluster randomization. Int $\mathcal{f}$ Epidemiol 1985; 14:322-6.

10 Wonnacott T, Wonnacott R. Econometrics. New York: Wiley, 